M. Hashimoto

Nagoya Math. J.

Vol. 186 (2007), 165-171

\title{
BASE CHANGE OF INVARIANT SUBRINGS
}

\author{
MITSUYASU HASHIMOTO
}

\begin{abstract}
Let $R$ be a Dedekind domain, $G$ an affine flat $R$-group scheme, and $B$ a flat $R$-algebra on which $G$ acts. Let $A \rightarrow B^{G}$ be an $R$-algebra map. Assume that $A$ is Noetherian. We show that if the induced map $K \otimes A \rightarrow(K \otimes B)^{K \otimes G}$ is an isomorphism for any algebraically closed field $K$ which is an $R$-algebra, then $S \otimes A \rightarrow(S \otimes B)^{S \otimes G}$ is an isomorphism for any $R$-algebra $S$.
\end{abstract}

\section{$\S 1$. Introduction}

In this paper, we prove the following.

Theorem 1. Let $R$ be a Dedekind domain, $G$ an affine flat $R$-group scheme, and $M$ an $R$-flat $G$-module. Let $A$ be a Noetherian $R$-algebra, and $V$ a finitely generated $A$-module. Let $\varphi: V \rightarrow M^{G}$ be an R-linear map. If the induced map $\varphi_{K}: K \otimes V \rightarrow(K \otimes M)^{K \otimes G}$ is an isomorphism for any algebraically closed field $K$ which is an $R$-algebra, then the canonical map $\varphi_{S}: S \otimes V \rightarrow(S \otimes M)^{S \otimes G}$ is an isomorphism for any $R$-algebra $S$.

As a corollary, we have the following.

Corollary 2. Let $R$ be a Dedekind domain, $G$ an affine flat $R$-group scheme, and $B$ a flat $R$-algebra on which $G$-acts. Let $A$ be a Noetherian $R$-algebra, and $\varphi: A \rightarrow B^{G}$ an $R$-algebra map. If the induced map $\varphi_{K}: K \otimes$ $A \rightarrow(K \otimes B)^{K \otimes G}$ is an isomorphism for any algebraically closed field $K$ which is an R-algebra, then the canonical map $\varphi_{S}: S \otimes A \rightarrow(S \otimes B)^{S \otimes G}$ is an isomorphism for any $R$-algebra $S$.

So we may work only over algebraically closed field instead of general commutative ring, once we know that the action and the candidate of the generator and the relation of the invariant subring are given over a Dedekind domain (e.g., $\mathbb{Z}$ ), and the group scheme in problem is flat over the Dedekind domain.

Received May 9, 2005.

2000 Mathematics Subject Classification: 13A50. 
De Concini and Procesi [3] calculated the invariant subrings for several important group scheme actions over an arbitrary commutative ring. In [6], a simple proof (for the action of the general linear group and the symplectic group) utilizing a geometric argument over a field is given. In order to reduce the case of general base ring to the case of base field, the knowledge of good filtrations is utilized in [6], but this was a completely general theory as above, since we know that the general linear group and the symplectic group are flat over $\mathbb{Z}$.

In Section 2, we prove the theorem above. In Section 3, we give an example of applications.

\section{$\S 2$. The proof of the main theorem}

Let $R$ be a commutative ring, and $G$ a flat $R$-group scheme. Let $C$ be the coordinate ring $R[G]$ of $G$. It is an $R$-flat commutative $R$ Hopf algebra. A $G$-module is nothing but a right $C$-comodule, see [7, Chapter 2]. For a $G$-module $M, M^{G}=\{m \in M \mid \omega(m)=m \otimes 1\}$, where $\omega: M \rightarrow M \otimes C$ is the coaction. By means of the natural inclusion $\operatorname{Hom}_{G}(R, M) \hookrightarrow \operatorname{Hom}_{R}(R, M)=M$, the $R$-module $\operatorname{Hom}_{G}(R, M)$ is identified with $M^{G}$, where $R$ is equipped with the trivial $G$-module structure.

In general, an $R$-module $V$ is considered as a trivial $G$-module. So, for a $G$-module $M$ and an $R$-module $V, V \otimes M$ is a $G$-module with the coaction

$$
1_{V} \otimes \omega_{M}: V \otimes M \rightarrow V \otimes M \otimes C
$$

where $\omega_{M}$ is the coaction of $M$.

The category of $G$-modules is abelian, with enough injectives, see [5, Lemma I.3.3.3] and [5, Lemma I.3.5.9]. For a $G$-module $M$ and an $R$-algebra $S$, the right $S \otimes C$-comodule structure of $S \otimes M$ is given by the composite

$$
S \otimes M \stackrel{1_{S} \otimes \omega}{\longrightarrow} S \otimes M \otimes C \stackrel{\alpha}{\longrightarrow}(S \otimes M) \otimes_{S}(S \otimes C),
$$

where $\alpha$ is the isomorphism given by $\alpha(s \otimes m \otimes c)=(s \otimes m) \otimes(1 \otimes c)$. In particular, $(S \otimes M)^{S \otimes G}=(S \otimes M)^{G}$. So $(S \otimes M)^{G}$ is an $S$-module.

Let $\varphi: V \rightarrow M^{G}$ be an $R$-linear map. Then we define $\varphi_{S}: S \otimes V \rightarrow$ $(S \otimes M)^{G}$ by $\varphi_{S}(s \otimes v)=s \otimes \varphi(v)$. For an $R$-algebra map $S \rightarrow S^{\prime}$, we define $\rho_{S^{\prime}, S}: S^{\prime} \otimes_{S}(S \otimes M)^{G} \rightarrow\left(S^{\prime} \otimes M\right)^{G}$ by

$$
\rho_{S^{\prime}, S}\left(s^{\prime} \otimes\left(\sum_{i} s_{i} \otimes m_{i}\right)\right)=\sum_{i} s^{\prime} s_{i} \otimes m_{i} .
$$


We denote $\rho_{S, R}: S \otimes M^{G} \rightarrow(S \otimes M)^{G}$ by $\rho_{S}$. So $\rho_{S}(s \otimes m)=s \otimes m$ for $s \in S$ and $m \in M^{G}$. Note that $\varphi_{S}$ is the composite

$$
S \otimes V \stackrel{1 \otimes \varphi}{\longrightarrow} S \otimes M^{G} \stackrel{\rho_{S}}{\longrightarrow}(S \otimes M)^{G} .
$$

For a $G$-module $M$, we denote $\operatorname{Ext}_{G}^{i}(R, M)$ by $H^{i}(G, M)$, and call it the $i$ th $G$-cohomology of $M$. In particular, $H^{0}(G, M)=M^{G}$.

Let $M$ be a $G$-module. Then by [5, Lemma I.3.6.16], $H^{i}(G, M) \cong$ $H^{i}\left(\operatorname{Cobar}_{C}(M, R)\right)$, where $\mathbb{F}(M):=\operatorname{Cobar}_{C}(M, R)$ is the complex

$$
M \stackrel{\delta^{0}}{\longrightarrow} M \otimes C \stackrel{\delta^{1}}{\longrightarrow} M \otimes C \otimes C \stackrel{\delta^{2}}{\longrightarrow} \cdots
$$

whose boundary map is given by

$$
\begin{aligned}
& \delta^{n}=(-1)^{n+1} \omega_{M} \otimes 1_{C^{\otimes n}}+\sum_{i=0}^{n-1}(-1)^{n-i} 1_{M} \otimes 1_{C \otimes i} \otimes \Delta_{C} \otimes 1_{C \otimes n-i-1} \\
& +1_{M} \otimes 1_{C^{\otimes n}} \otimes u,
\end{aligned}
$$

where $\Delta_{C}: C \rightarrow C \otimes C$ is the coproduct, and $u: R \rightarrow C$ is the unit map. By definition, for an $R$-module $V, \mathbb{F}(V \otimes M) \cong V \otimes \mathbb{F}(M)$. If $M$ is $R$-flat, then $\mathbb{F}(M)$ is an $R$-flat complex. By the universal coefficient theorem [5, Lemma III.2.1.2] and its proof, we have the following.

Lemma 3. If $R$ is a Dedekind domain and $M$ is an $R$-flat $G$-module, there is an exact sequence

$$
0 \rightarrow S \otimes M^{G} \stackrel{\rho_{S}}{\longrightarrow}(S \otimes M)^{G} \rightarrow \operatorname{Tor}_{1}^{R}\left(S, H^{1}(G, M)\right) \rightarrow 0 .
$$

Proof of Theorem 1. Let $R, G, A, V$, and $M$ be as in the theorem.

First, we prove the theorem for the case where $S$ is a field. Let $K$ be the algebraic closure of $S$. Taking the tensor product of $\varphi_{S}: S \otimes V \rightarrow(S \otimes M)^{G}$ with $K$ over $S$, we get $1 \otimes \varphi_{S}: K \otimes V \rightarrow K \otimes_{S}(S \otimes M)^{G}$. As $K$ is faithfully flat over $S$, it suffices to show that this map is an isomorphism. The composite

$$
K \otimes V \stackrel{1 \otimes \varphi_{S}}{\longrightarrow} K \otimes S(S \otimes M)^{G} \stackrel{\rho_{K, S}}{\longrightarrow}(K \otimes M)^{G}
$$

is $\varphi_{K}$, which is an isomorphism. Since $K$ is $S$-flat, $\rho_{K, S}$ is an isomorphism by Lemma 3. So $1 \otimes \varphi_{S}$ is an isomorphism as desired, and the theorem is true for the case that $S$ is a field. 
Next, we show that $H^{1}(G, M)$ is $R$-flat. Since $R$ is Noetherian, it suffices to show that $\operatorname{Tor}_{1}^{R}\left(R / P, H^{1}(G, M)\right)=0$ for any prime ideal $P$ of $R$. Since $R$ is a one dimensional domain, it suffices to show that $\operatorname{Tor}_{1}^{R}\left(R / \mathfrak{m}, H^{1}(G, M)\right)=0$ for any maximal ideal $\mathfrak{m}$ of $R$. On the other hand, $\varphi_{R / \mathfrak{m}}$, which is the composite

$$
R / \mathfrak{m} \otimes V \stackrel{1 \otimes \varphi}{\longrightarrow} R / \mathfrak{m} \otimes M^{G} \stackrel{\rho_{R / \mathfrak{m}}}{\longrightarrow}(R / \mathfrak{m} \otimes M)^{G},
$$

is an isomorphism by the last paragraph. So $\rho_{R / \mathfrak{m}}$ is surjective. By Lemma 3, $\operatorname{Tor}_{1}^{R}\left(R / \mathfrak{m}, H^{1}(G, M)\right)=0$. Hence $H^{1}(G, M)$ is $R$-flat, as desired.

Since $H^{1}(G, M)$ is $R$-flat,

$$
\rho_{S}: S \otimes M^{G} \rightarrow(S \otimes M)^{G}
$$

is an isomorphism for any $R$-algebra $S$ by Lemma 3 . Since the composite

$$
K \otimes V \stackrel{1 \otimes \varphi}{\longrightarrow} K \otimes M^{G} \stackrel{\rho_{K}}{\longrightarrow}(K \otimes M)^{G},
$$

which agrees with $\varphi_{K}$, is an isomorphism and $\rho_{K}$ is also an isomorphism for any field $K$ which is an $R$-algebra, we have that $1 \otimes \varphi: K \otimes V \rightarrow K \otimes M^{G}$ is an isomorphism.

Next, we show that $V$ is $R$-flat. First, we prove this for the case that $R$ is a DVR. Let $t$ be a generator of the maximal ideal of $R$. Since $V$ is a Noetherian $A$-module, the torsion part $V_{\text {tor }}=\bigcup_{r>0}\left(0: V t^{r}\right)$ as an $R$-module agrees with $\left(0:_{V} t^{r}\right)$ for some $r$. Assume that $V_{\text {tor }} \neq 0$ for a contradiction. Then $r \geq 1$. We take $r$ as small as possible. Take $a \in\left(0: t^{r}\right) \backslash\left(0: t^{r-1}\right)$. If $a \in t V$, then $a=t a^{\prime}$ for some $a^{\prime} \in V$. Then $a^{\prime} \in V_{\text {tor }}=\left(0: t^{r}\right)$. So $t^{r-1} a=t^{r} a^{\prime}=0$. This contradicts the choice of $a$. So $a \notin t V$. Thus $1 \otimes a \in R / t R \otimes_{R} V$ is nonzero. Since $1 \otimes \varphi: R / t R \otimes_{R} V \rightarrow R / t R \otimes M^{G}$ is an isomorphism, $1 \otimes \varphi(a) \in R / t R \otimes M^{G}$ is nonzero. This shows that $\varphi(a) \neq 0$ in $M^{G}$. Since $M^{G}$ is a torsion free $R$-module, $\varphi\left(t^{r} a\right)=t^{r} \varphi(a)$ is nonzero. This contradicts the assumption $t^{r} a=0$. Hence $V$ is $R$-torsion free. Since $R$ is a DVR, $V$ is $R$-flat. Now consider the general case. Let $\mathfrak{m}$ be a maximal ideal of $R$. Applying the discussion above to $R^{\prime}=R_{\mathfrak{m}}$, $A^{\prime}=R^{\prime} \otimes A, V^{\prime}=R^{\prime} \otimes V$ and $M^{\prime}=R^{\prime} \otimes M$, we have that $V_{\mathfrak{m}}$ is $R_{\mathfrak{m}}$-flat for any $\mathfrak{m}$. This shows that $V$ is $R$-flat.

By [5, Lemma I.2.1.4], $\varphi: V \rightarrow M^{G}$ is injective, and $C:=\operatorname{Coker} \varphi$ is $R$-flat. Since $K \otimes C=0$ for any field $K$ which is an $R$-algebra, we have that $C=0$ by [5, Corollary I.2.1.6]. Hence $\varphi$ is an isomorphism. 
Let $S$ be any $R$-algebra. The composite

$$
S \otimes V \stackrel{1 \otimes \varphi}{\longrightarrow} S \otimes M^{G} \stackrel{\rho_{S}}{\longrightarrow}(S \otimes M)^{G}
$$

is an isomorphism, since $1 \otimes \varphi$ and $\rho_{S}$ are. This is what we wanted to prove.

\section{$\S 3$. An application}

Let $R$ be a commutative ring. For an $R$-scheme $Z$, we denote $\Gamma\left(Z, \mathcal{O}_{Z}\right)$ by $R[Z]$. For $v \geq 0$ and finite free $R$-modules $F$ and $G$, we denote by $Y_{v}(F, G)$ the closed subscheme of $\operatorname{Hom}_{R}(F, G)$ consisting of $R$-linear maps of rank at most $v$. We denote the kernel of the map $R\left[\operatorname{Hom}_{R}(F, G)\right] \rightarrow$ $R\left[Y_{v}(F, G)\right]$ by $I_{v+1}(F, G)$. If $F$ and $G$ are of rank $f$ and $g$, respectively, then $R\left[\operatorname{Hom}_{R}(F, G)\right]$ is identified with the polynomial $\operatorname{ring} R\left[x_{i j}\right]_{1 \leq i \leq g, 1 \leq j \leq f}$ in $f g$ variables, and $I_{v+1}(F, G)$ is identified with the ideal of $R\left[x_{i j}\right]$ generated by the all $(v+1)$-minors of the matrix $\left(x_{i j}\right)$. Note that if $v \geq \min (f, g)$, then $Y_{v}(F, G)=\operatorname{Hom}_{R}(F, G)$, and $I_{v+1}(F, G)=0$.

Let $m, n, r, s, t \in \mathbb{Z}_{\geq 0}$ such that $s \leq m, r$ and $t \leq n, r$. Set $u:=\min (s, t)$. Let $V:=R^{n}, W:=R^{m}, E:=R^{r}, X:=Y_{s}(E, W) \times Y_{t}(V, E)$, and $Y:=$ $Y_{u}(V, W)$. We define $\pi: X \rightarrow Y$ by $\pi(\varphi, \psi)=\varphi \circ \psi$. Let $G:=G L(E)$ and $G^{\prime}:=G L(W) \times G L(V)$. Then $G \times G^{\prime}$ acts on $X$ by $\left(g,\left(g_{1}, g_{2}\right)\right) \cdot(\varphi, \psi)=$ $\left(g_{1} \varphi g^{-1}, g \psi g_{2}^{-1}\right)$ for $g \in G, g_{1} \in G L(W), g_{2} \in G L(V), \varphi \in Y_{s}(E, W)$, and $\psi \in Y_{t}(V, E)$. Letting $G \times G^{\prime}$ act on $Y$ by $\left(g,\left(g_{1}, g_{2}\right)\right) \cdot \rho=g_{1} \rho g_{2}^{-1}$ for $g \in G$, $g_{1} \in G L(W), g_{2} \in G L(V)$, and $\rho \in Y$, the morphism $\pi$ is $G \times G^{\prime}$-equivariant. Note that $G$ acts on $Y$ trivially.

As an application of Theorem 1, we prove the following.

THEOREM 4. The morphism $\pi: X \rightarrow Y$ induces an isomorphism $\pi^{\#}: R[Y] \rightarrow R[X]^{G}$.

Proof. By Theorem 1, we may assume that $R=K$ is an algebraically closed field.

Let us recall some basic facts from representation theory. A $G$-module $M$ is said to have good filtrations if $\operatorname{Ext}_{G}^{1}\left(\Delta_{G}(\lambda), M\right)=0$ for any dominant weight $\lambda$, where $\Delta_{G}(\lambda)$ denotes the Weyl module of highest weight $\lambda$, see $[7,($ II.4.16)].

For a partition $\lambda=\left(\lambda_{1}, \ldots, \lambda_{k}\right)$ with $\lambda_{1} \leq r$, the Schur module [1] $L_{\lambda} E^{*}$ is a dual Weyl module. In fact, $L_{\lambda} E^{*} \cong\left(\bigwedge^{r} E^{*}\right)^{\otimes k} \otimes L_{\mu} E$, where $\mu=(r-$ $\left.\lambda_{k}, \ldots, r-\lambda_{1}\right)$. By the Cauchy formula [1], $K[\operatorname{Hom}(E, W)] \cong \operatorname{Sym}\left(E \otimes W^{*}\right)$, 
$I_{s+1}(E, W), K\left[Y_{s}(E, W)\right], K[\operatorname{Hom}(V, E)] \cong \operatorname{Sym}\left(V \otimes E^{*}\right), I_{t+1}(V, E)$, and $K\left[Y_{t}(V, E)\right]$ have good filtrations as $G$-modules. Since modules with good filtrations are closed under tensor products [9], [4], [8] and extensions, the kernel $I$ of the canonical surjective map

$$
\rho: K[\operatorname{Hom}(E, W) \times \operatorname{Hom}(V, E)] \rightarrow K\left[Y_{s}(E, W) \times Y_{t}(V, E)\right]
$$

has good filtrations, since there is a short exact sequence

$$
0 \rightarrow I_{s+1}(E, W) \otimes K[\operatorname{Hom}(V, E)] \rightarrow I \rightarrow K\left[Y_{s}(E, W)\right] \otimes I_{t+1}(V, E) \rightarrow 0 .
$$

Hence $H^{1}(G, I)=0$. It follows that $\rho$ induces a surjective map

$$
\rho^{G}: K[\operatorname{Hom}(E, W) \times \operatorname{Hom}(V, E)]^{G} \rightarrow K\left[Y_{s}(E, W) \times Y_{t}(V, E)\right]^{G}=K[X]^{G} .
$$

By the following theorem due to De Concini and Procesi [3], $\pi^{\#}: K[Y] \rightarrow$ $K[X]^{G}$ is surjective.

TheOrem 5. The composition

$$
\operatorname{Hom}(E, W) \times \operatorname{Hom}(V, E) \rightarrow Y_{r}(V, W) \quad((\varphi, \psi) \mapsto \varphi \psi)
$$

induces an isomorphism $K\left[Y_{r}(V, W)\right] \rightarrow K[\operatorname{Hom}(E, W) \times \operatorname{Hom}(V, E)]^{G}$.

It remains to prove that $\pi^{\#}: K[Y] \rightarrow K[X]^{G}$ is injective. As we know that $K[Y]$ is an integral domain (see e.g., $[2,(6.3)]$ ), it suffices to show that $\pi$ is dominating. By linear algebra, for each $i$ such that $0 \leq i \leq u$, the set of linear maps $V \rightarrow W$ of rank $i$ forms one $G^{\prime}$-orbit. Moreover, the $G^{\prime}$-orbit of rank $u$ linear maps is dense in $Y$. Since $\pi$ is $G^{\prime}$-invariant, it suffices to show that $\pi(X)$ contains at least one linear map of rank $u$. But this is trivial.

\section{REFERENCES}

[1] K. Akin, D. A. Buchsbaum and J. Weyman, Schur functors and Schur complexes, Adv. Math., 44 (1982), 207-278.

[2] W. Bruns and U. Vetter, Determinantal Rings, Lecture Notes in Math. 1327, Springer, 1988.

[3] C. De Concini and C. Procesi, A characteristic free approach to invariant theory, Adv. Math., 21 (1976), 330-354.

[4] S. Donkin, Rational Representations of Algebraic Groups, Lecture Notes in Math. 1140, Springer, 1985. 
[5] M. Hashimoto, Auslander-Buchweitz Approximations of Equivariant Modules, Cambridge, 2000.

[6] M. Hashimoto, Another proof of theorems of De Concini and Procesi, to appear in J. Math. Kyoto Univ.

[7] J. C. Jantzen, Representations of Algebraic Groups, 2nd edition, AMS, 2003.

[8] O. Mathieu, Filtrations of G-modules, Ann. Sci. École Norm. Sup. (4), 23 (1990), 625-644.

[9] J. Wang, Sheaf cohomology on $G / B$ and tensor products of Weyl modules, J. Algebra, 77 (1982), 162-185.

Graduate School of Mathematics

Nagoya University

Chikusa-ku, Nagoya 464-8602

Japan

hasimoto@math.nagoya-u.ac.jp 